\title{
Trismus following different treatment modalities for head and neck cancer: a systematic review of subjective measures
}

\author{
Sook Y. Loh ${ }^{1} \cdot$ Robert W. J. Mcleod ${ }^{2} \cdot$ Hassan A. Elhassan ${ }^{2}$
}

Received: 12 November 2016 / Accepted: 21 February 2017 / Published online: 25 March 2017

(c) The Author(s) 2017. This article is an open access publication

\begin{abstract}
The aim of this review was to compare systematically the subjective measure of trismus between different interventions to treat head and neck cancer, particularly those of the oropharynx. Using The Preferred Reporting Items for Systematic Review and Meta-Analyses (PRISMA) Guidelines, Six databases were searched for the text using various terms which include "oropharyngeal/ head and neck cancer", "trismus/mouth opening" and the various treatment modalities. Included in the review were clinical studies ( $>$ or $=10$ patients). Three observers independently assessed the papers identified. Among the six studies reviewed, five showed a significantly worst outcome with regard to the quality-of-life questionnaire scores for a radiotherapy or surgery and radiotherapy $(\mathrm{RT}) \pm$ chemotherapy or chemoradiotherapy when compared to surgery alone. Only one study showed no significant difference between surgery alone and other treatment modalities. Subjective quality-of-life measures are a concurrent part of modern surgical practice. Although subjective measures were utilised to measure post operative trismus successfully, there was no consensus as to which treatment modality had overall better outcomes, with conflicting studies in keeping with the current debate in this field. Larger and higher quality studies are needed to compare all three treatment modalities.
\end{abstract}

Keywords Trismus - Oropharyngeal carcinoma · Surgery $\cdot$ Radiotherapy $\cdot$ Chemotherapy $\cdot$ Quality of life

Sook Y. Loh

LohSY@ cardiff.ac.uk

Cardiff University School of Medicine, Cardiff, UK

2 Department of Otolaryngology, University Hospital of Wales, Cardiff, UK

\section{Introduction}

Cancers of the upper aerodigestive tract, collectively known as head and neck cancers, arise from a myriad of sites, including the oral cavity, pharynx, and larynx, as well as the nasal cavity and sinuses [1-3]. Oropharyngeal cancer (OPC) is a rare cancer overall but common in the head and neck region [4]. Head and neck cancer is the sixth most common type of cancer, making up 5.3\% of all cases and account for an estimated 348,300 new cancer cases and 179,600 cancer deaths worldwide per year [5]. The median age for diagnosis is around mid-60s, with male predominance, especially for laryngeal cancer [6]. Squamous cell carcinomas (SCC) of varying degrees of differentiation make up the majority of head and neck cancers $[2,3]$. About two-thirds of these SCC patients present at an advanced stage, usually with nodal involvement [3].

There is wide discrepancy in treating head and neck cancer. In recent decades, there has been a tendency towards organ preserving treatment [2] and as most head and neck cancers respond well to radiotherapy (at least in the first instance), surgery can be avoided in a high proportion of cases [2]. Chemotherapy has accentuated this organ preserving approach [2]. National Comprehensive Cancer Network (NCCN) guidelines consider concurrent chemoradiation therapy (CCRT) as first-line treatment for oropharyngeal cancers; however, the evidence supporting this is equivocal [7].

All treatment modalities have their pros and cons. Surgery may result in loss of function associated with excision of anatomical structures, whereas radiotherapy may be accompanied by debilitating dysphagia and long-term loss of oral lubrication [2]. Radiotherapy induces fibrosis in the muscles of mastication, leading to trismus, as well as necrosis of bone and soft tissue which restricts mouth 
opening $[8,9]$. Chemotherapy too is associated with significant morbidity and can cause renal dysfunction, ototoxicity, and myelosuppression [2]. Decisions on treatment modality depend on the tumour site and stage, expected functional outcomes and the patients comorbidities and ability to tolerate treatment [3]. There is currently no consensus as to which treatment modality results in better outcomes.

Trismus, which is restricted mouth opening, is common in head and neck cancer patients and interferes with activities, such as eating, swallowing, and speaking $[10,11]$. It also interferes with oral hygiene and can be particularly discomforting to patients [12]. Trismus may be caused by tumour infiltration into the masticatory muscles, specifically the pterygoids, or temporomandibular joint (TMJ) or induced by cancer treatment, including surgery and/or radiotherapy(RT) $[13,14]$. Post-treatment trismus is unpredictable in both its frequency and severity [12] and usually develops 3-6 month post-radiotherapy and often becomes a lifelong problem [13].

Prevention of trismus is more desirable than treating it [14]. Patients at risk of trismus should have home exercises to maintain maximum mouth opening and jaw mobility as soon as they start radiotherapy $[15,16]$. Patients who develop trismus require an intensive exercise programme, and if necessary, combined with physiotherapy to improve mouth opening [15]. Prosthetic appliances (dynamic bite openers) containing springs and bands are able to re-stretch the muscles to help patients suffering from trismus [15, 16]. Patient concordance with trismus exercises is paramount if preventative and treatment regimes are to be successful [17].

\section{Review aim}

Trismus can be measured both objectively and subjectively. The goal of this review is to compare the different tools for the subjective measures of trismus and assess the most suitable questionnaire to be used for measuring trismus. We include a systematic literature review to compare the effect of different head and neck cancer treatment modalities on trismus using subjective measures encountered.

\section{Objective measures of trismus}

Normal mouth opening varies between individuals, within a range of 40-60 $\mathrm{mm}$ [18]. Males generally display greater mouth opening than females [19]. Maximal interincisal opening (MIO) is the maximal distance between the edges of the upper and lower incisors and is widely used to objectively measure trismus [8, 10, 11, 20-23]. For edentulous patients, the distance from one alveolar ridge to the opposing side vertically can be used instead [14]. These measurements can be made using callipers or other devices $[11,20]$. The MIO should be measured before treatment is commenced, and the patient or clinician should measure this distance frequently post-treatment to ensure its maintenance [17]. As there is no agreed absolute measurement of trismus in clinical practice, studies have mostly used a cutoff of $\leq 35 \mathrm{~mm}[10,11,21-26]$.

\section{Subjective measure of trismus}

Patients may continue to experience trismus in spite of objectively 'normal' MIO. The emergence of the patient reported outcome measures (PROMs) and quality-of-life questionnaires allows clinicians to look beyond mortality as the sole outcome measure of successful medical interventions. Subjective measures can also be used to compare different treatments [27]. Such subjective measures of trismus are vital in managing this condition.

Our review identified several questionnaires that address trismus (Table 1). These subjective trismus measures consist of either a mouth opening specific questionnaires, such as the Mandibular Function Impairment Questionnaire (MFIQ) [21], or a subset question in a general cancer quality-of-life questionnaire, such as the Performance Status Scale (PSS) [28].

Table 1 Summary of questionnaires

\begin{tabular}{lc}
\hline Mouth-opening specific questionnaires & General quality-of-life questionnaires \\
\hline Mandibular Function Impairment Questionnaire (MFIQ) [21] & European Organization for Research and Treatment of Cancer (EORTC) \\
& QLQ-C30 questionnaire [7, 8, 23, 24, 27, 29-32] \\
The Liverpool Oral Rehabilitation Questionnaire (LORQ v3) [26, 33] & EORTC QLQ-H\&N35 questionnaire [7, 8, 23, 24, 27, 29-32] \\
Gothenburg Trismus Questionnaire (GTQ) [20] & Performance Status Scale (PSS) [28] \\
& The University of Washington quality-of-life scale (UWQOL v4) [23, \\
& 26] \\
& Functional Assessment of Cancer Therapy-Head and Neck Scale \\
& (FACT-H\&N) [34] \\
\hline
\end{tabular}


There is a suggested definition of severe trismus with a PSS score of $\leq 50$, European Organization for Research and Treatment of Cancer (EORTC) QLQ-C30 and EORTC QLQ-H\&N35 $\geq 50$ [28].

\section{Mouth-opening specific questionnaires}

The first study that aimed to determine the cutoff for trismus used the Mandibular Function Impairment Questionnaire (MFIQ) [21]. This questionnaire contains 11 items which assessed perceived difficulties in mandibular function during social activities, speaking, taking large bites, chewing hard food, chewing soft food, work and/or daily activities, drinking, laughing, chewing resistant food, yawning, and kissing [21]. There were also six other items which took into account difficulties during eating specific foods, i.e., a hard cookie, meat, a raw carrot, French bread, peanuts/almond, and an apple [21]. Answers were scored (0) no difficulty, (1) a little difficulty, (2) quite a bit of difficulty, (3) much difficulty, and (4) very great difficulty or impossible without help [21]. Scores were summed up, with a range between 0 and 68 and a high score indicating more impairment [21]. The internal consistency of the questionnaire lies between 0.80 and 0.95 [29].

The Liverpool Oral Rehabilitation Questionnaire (LORQ v3) comprises 40 items, whereby 17 relate to oral function, oro-facial appearance, and social interaction [30]. Five questions were included to assess the impact of chewing ability on social life and choice of food: (1) did you experience difficulty with chewing? (2) did you have pain when you chew? (3) did your chewing ability affect your social life? (4) did your chewing ability influence your choice of foods? and (5) did you experience difficulty with mouth opening? [26] Options for an answer included 'Always', 'Often', 'Sometimes', or 'Never' [26].

The Gothenburg Trismus Questionnaire (GTQ) is a trismus specific questionnaire with good psychometric properties (validity and reliability), but has only been used in one study [20]. This questionnaire contains 21 items with 13 items divided into the three domains: jaw-related problems (six items); eating limitations (four items); and muscular tension (three items) [20]. The domains and single items range from 0 to $100 ; 100$ indicating maximal amount of symptoms and 0 being symptom free [20]. The GTQ has a 1 week recall period for the three domains [20]. Patients with trismus reported more health-related quality-of-life impairments in the domains of mouth opening $(p<0.001)$, jaw-related problems $(p<0.05)$, eating limitations $(p<0.05)$, and muscular tension $(p<0.001)$ [20]. These results were in line with the incidence of trismus, and are compatible with results from other studies $[8,20$, 31]. The GTQ has been suggested as a screening tool and for evaluating endpoints in intervention for jaw physiotherapy and rehabilitation studies [20].

\section{General quality-of-life questionnaires}

The PSS consists of three subscales, such as eating in public, normalcy of diet, and intelligibility of patients speech $[28,32]$. Each is rated from 0 to 100 , whereby higher scores suggest better performance [32].

The UWQOL v4 is validated in the field of head and neck cancers but is not as widely used as the EORTC questionnaires [26]. It assesses 12 domains: pain, appearance, activity, recreation, swallowing, chewing, speech, shoulder function, taste, saliva, mood, and anxiety, whereby the chewing, saliva, mood, and anxiety domains which are measured on a Likert scale from 0 (worst) to 100 (best) were included in the study questionnaire to identify the effects of limited mouth opening on these domains [26]. There was no mention of a scoring system to define trismus using the UWQOL [26].

The FACT uses means of self-reporting and comprises 28 general +11 head and neck specific items, rated from 0 to 4 on a Likert-type scale [32]. The FACT domains describe function in six areas: physical well-being, social and family well-being, relationship with doctor, emotional well-being, functional well-being, and head- and neckrelated symptoms (HNS) [32, 33]. The FACT-H\&N module includes additional concerns, such as oral comfort, breathing, voice, eating, appearance, tobacco, alcohol, and communication [33].

The EORTC developed an established system assessing the health related quality of life (QOL) of head and neck cancer patients using two questionnaires, the general EORTC QLQ-C30 questionnaire, and the head- and neckspecific EORTC QLQ-H\&N35 module which subjectively measured mouth opening among the six scales [34]. The EORTC QLQ-C-30 is a self-assessment of health-related QOL for patients with cancer, including difference function scores, a score for global QOL, and symptoms scores relevant for cancer patients [28, 35]. The five functional scales are physical, role, emotional, cognitive, and social functioning, while the six symptom scores include dyspnoea, insomnia, appetite loss, constipation, diarrhoea, and financial difficulties [28]. The supplementary EORTC QLQ$\mathrm{H} \& \mathrm{~N} 35$ consists of 35 additional questions to assess head and neck cancer-related symptoms ( 7 multi-item scales and 11 single items, validated in a sample of 500 patients from Norway, Sweden, and The Netherlands) [28, 35, 36]. Scales and single questions are scored on categorical scales and linearly converted to a scale of 0-100 [35]. A score of 100 on the functioning scales and the Global QOL scale represents maximal functioning, while a score of 100 on the 
symptom scales and single items indicate worst possible symptoms [20]. Changes in the score of $>10$ points over time could be clinically significant [20]. The multi-item scales consist of pain, swallowing, senses, speech, social eating, social contact, and sexuality, while the single items are teeth, mouth opening, dry mouth, sticky saliva, coughing, feeling ill, pain killers, nutritional supplements, feeding tube, weight loss, and weight gain [28]. High function scores and a low symptom scores correlate to good functioning and few symptoms [35]. The EORTC has been used worldwide, including a validated Chinese version of the EORTC core questionnaire and head and neck module [37]. These questionnaires have been continuously re-evaluated and proven to be sensitive and comprehensive [38, 39].

\section{Correlation between subjective and objective trismus measures}

A couple of studies used both objective and subjective measurements for trismus [20, 23, 26]. Correlation between objective measurements of mouth opening and subjective measures using the UWQOL questionnaire has been attempted. This identified a significant association of mouth opening on the UWQOL chewing domain $\left(r_{\mathrm{s}}=0.45, p<0.0001\right)$ and in the UWQOL overall quality of life $\left(r_{\mathrm{s}}=0.25, p=0.01\right)$ [26]. However, there were exceptions whereby 16 patients with objective trismus denied problems with mouth opening while ten patients who were not shown clinically to have trismus complained subjectively of problems with mouth opening [26]. Despite this, correlation between subjective and objective measurements was overall strong.

Other studies that used subjective or objective measures have had comparable finding. Studies using objective measures found that patients who received adjuvant radiotherapy and multi-modality treatments had worst outcomes than those treated with surgery alone, in line with studies measuring trismus subjectively $[8,22,25,26,40,41]$. The difference in mean MFIQ scores for patients with trismus and without trismus for a cut-off point of $\leq 35 \mathrm{~mm}$ was significant [21]. An MFIQ score of 8.3 was the minimum score of for trismus, equivalent to a mouth opening of $35 \mathrm{~mm}$ [21].

\section{Materials and methods}

\section{Search strategy}

The preferred reporting items for systematic review and meta-analyses (PRISMA) guidelines were used to perform a systematic review of available literature. Studies evaluating the effects of different types of head and neck cancers, especially the oropharyngeal region on mouth opening, were searched for using PubMed, Cochrane Library, Science Direct, Scopus, EMBASE (1947-Present) and Ovid MEDLINE (1946 to May Week 2 2015). Four search domains were used and combined using "AND", while terms within each domain combined by "AND/OR". The keywords "head and neck cancer", "SCC oropharynx", "oropharyngeal cancer", "oropharyngeal carcinoma", "SCC base of tongue", "tonsil SCC", "oropharyngeal neoplasm", "oropharyngeal tumor", "oropharyngeal tumour", and "cancer of the oropharynx" were used in the first domain. The second domain encompassed "mouth opening", "jaw opening", and "trismus". Finally, the terms "surgery", "radiotherapy", "radiation therapy", "chemotherapy", "chemoradiotherapy", "chemoradiation", "chemotherapy and radiotherapy", "chemotherapy and radiation therapy", and "transoral robotic surgery" were used in the third domain. The final domain consisted of "quality of life".

The primary search identified 3243 records. 3146 of these were excluded following screening. 59 of the remaining studies were duplicates, thus leaving 38 studies for full text evaluation. Eight studies were added through cross-referencing. Following application of our inclusion and exclusion criteria to these full texts, a further 11 studies were excluded. A total of 35 studies were included, 19 involving subjective measures of trismus, while the other 16 only measured trismus objectively. Six subjective studies, which compared treatment modalities, were chosen for the final review (Fig. 1).

\section{Inclusion and exclusion criteria}

Original journal articles that studied the effects of oropharyngeal carcinoma treatment on mouth opening as assessed using qualitative measurements were included. Eligible, studies needed to be carried out on humans, involve ten or more patients, included post-treatment measurements, and included treatment with surgery, radiotherapy (RT), chemotherapy, or a combination of any of the three modalities. Articles were excluded if there was no patient involvement, unrelated to head and neck oncology, written in languages other than English, and focused on dental hygiene during oncology treatment or primarily discussed exercise regimes to treat trismus. Letters to the editor, case reports/series ( $<10$ participants) and poster abstracts were also excluded.

\section{Results}

The questionnaires used in the studies included in the systematic review were the EORTC QLQ-C30 and EORTC 
Fig. 1 Trismus search strategy to obtain literature using PRISMA guidelines
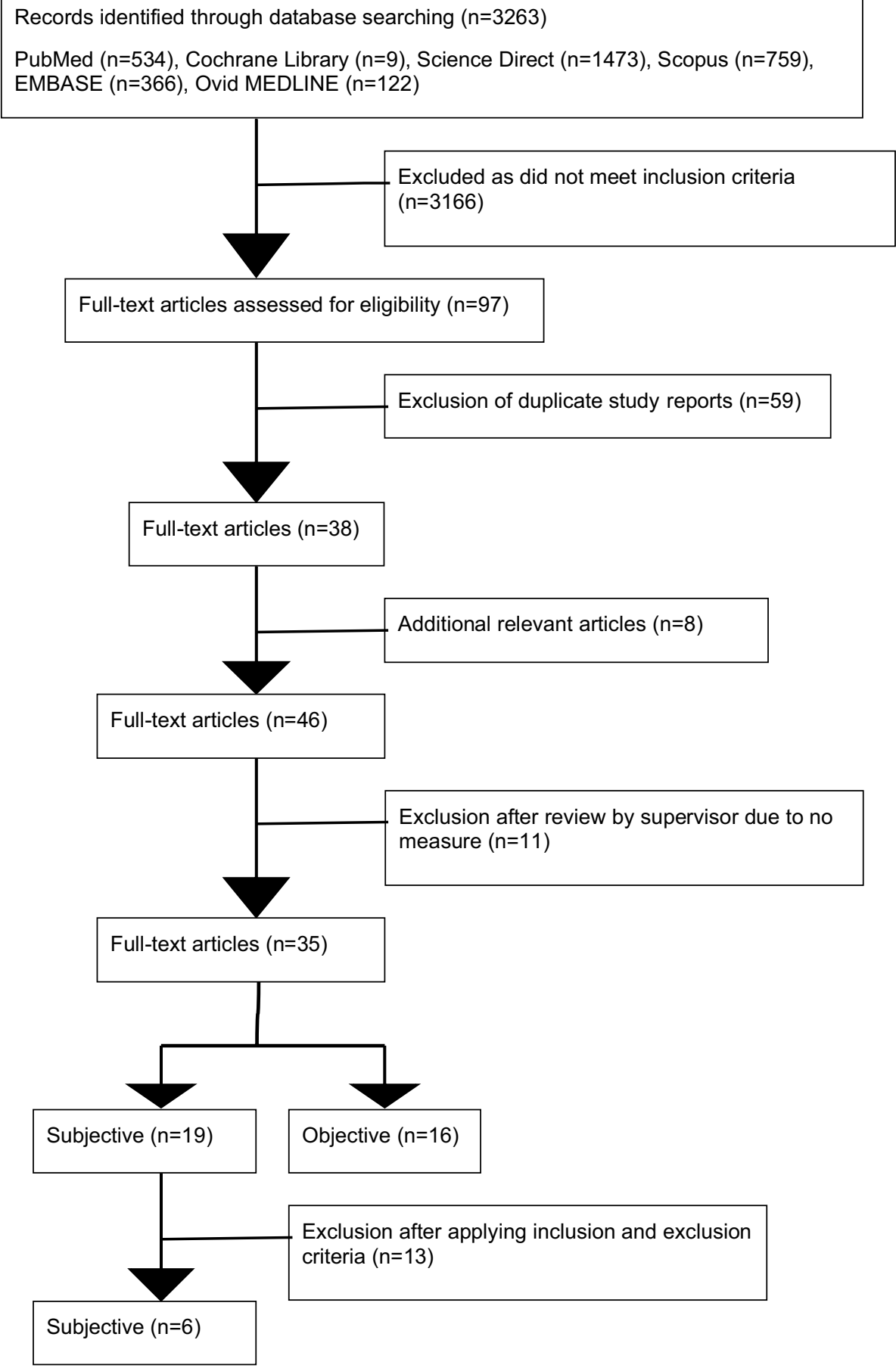

QLQ-H\&N35 [7, 27, 42-45]. None of these studies used objective measures to measure trismus. Table 2 shows a summary of the studies that were included in this review.

Ryzek et al. showed significantly better results among early stage oropharyngeal cancer (OPC) for the surgeryonly treatment group when compared to either surgery combined with RT or surgery combined with any type of adjuvant therapy for mouth opening $(p \leq 0.05)$ in the EORTC-QLQ-HN35 [27].
A further study evaluating OPC patients using the EORTC QLQ-H\&N35 questionnaire [43] found that radiation therapy significantly augmented the patient's complaint about restricted mouth opening (surgery versus radiation, $p=0.008$; surgery versus surgery plus irradiation, $p=0.0008$ ) [43]. When patients undergoing surgery $\pm \mathrm{RT}$ were compared with the non-surgically treated group (radiation therapy alone), patients in the no surgery group suffered significantly more from restricted mouth opening 


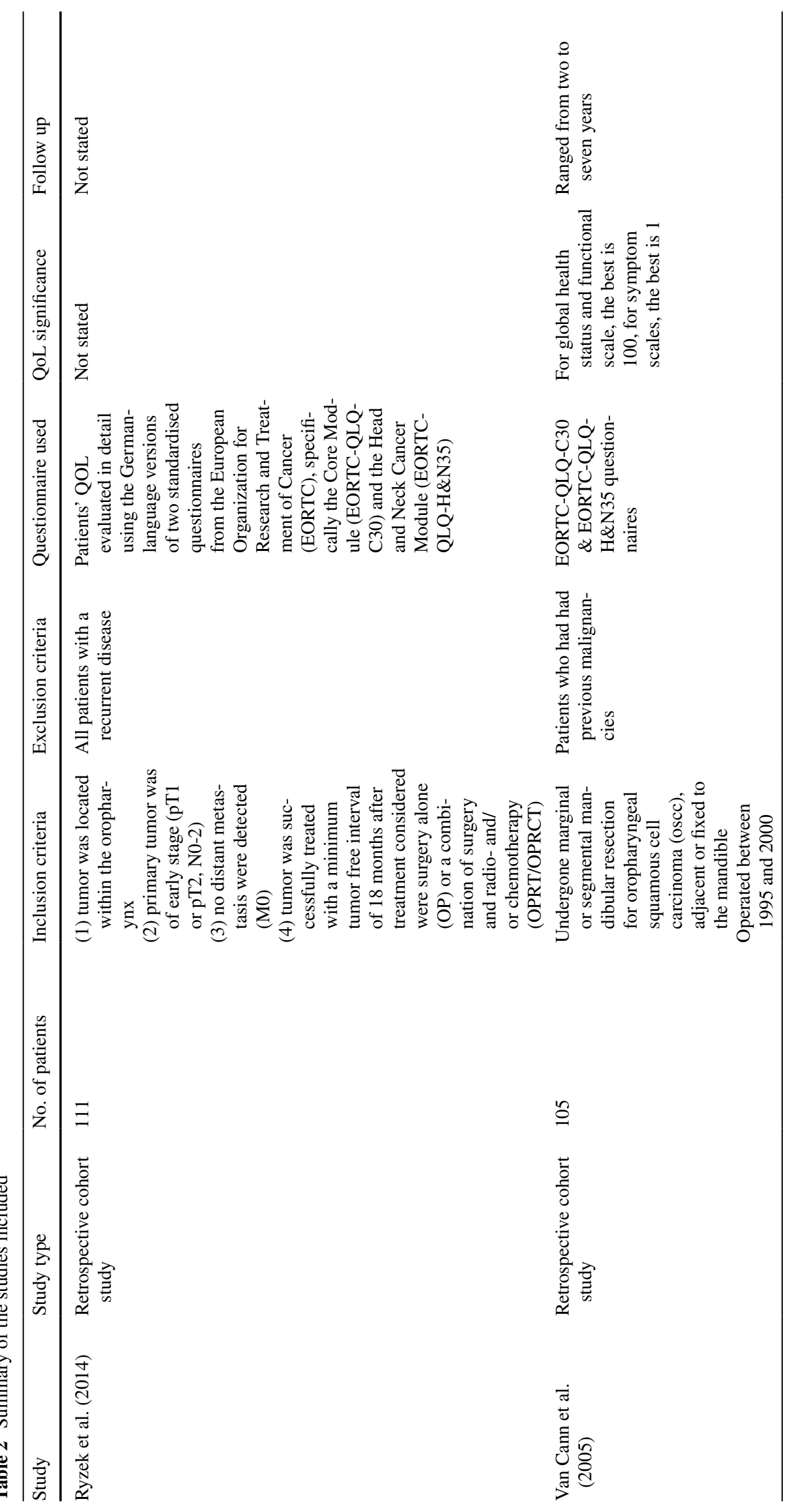




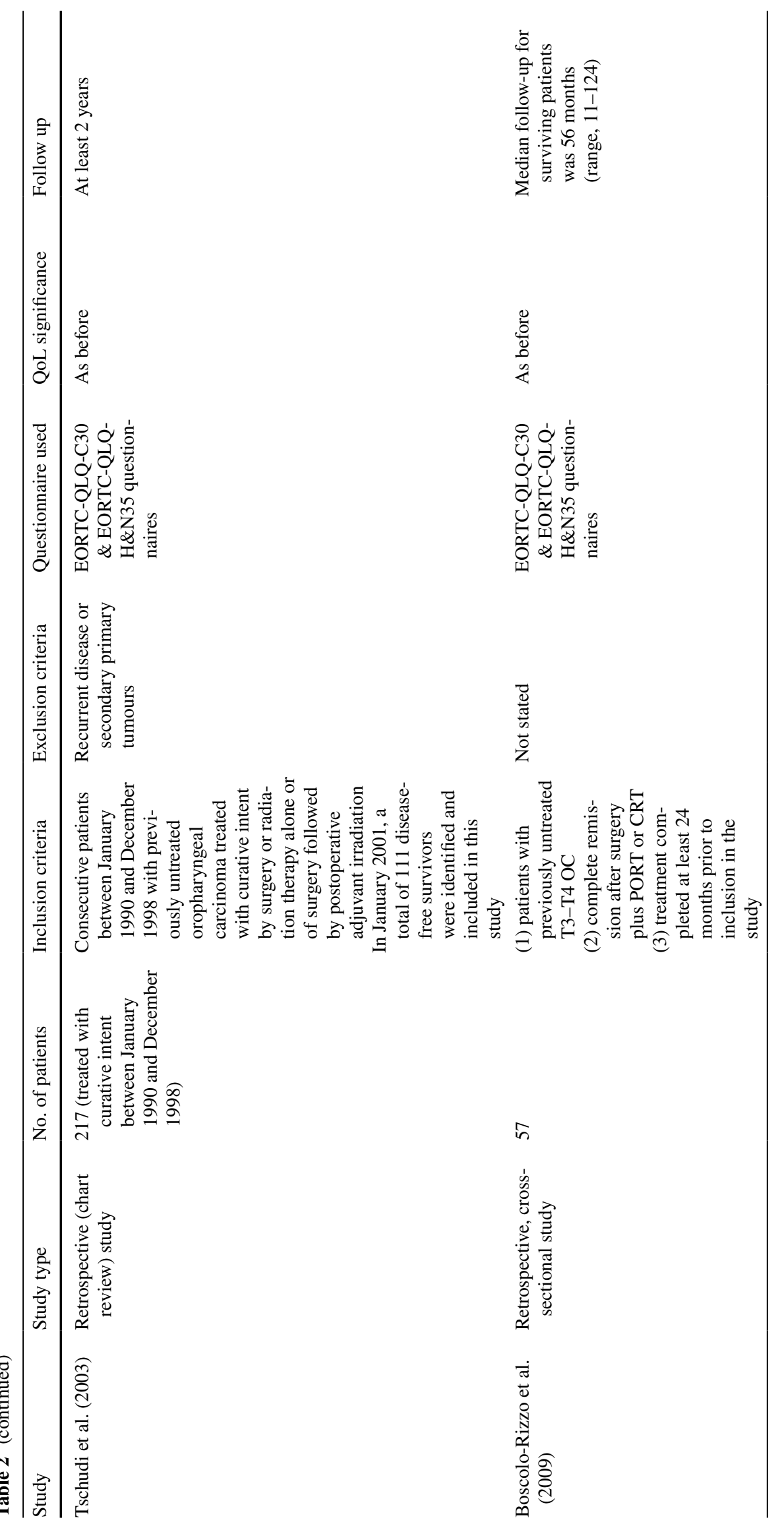




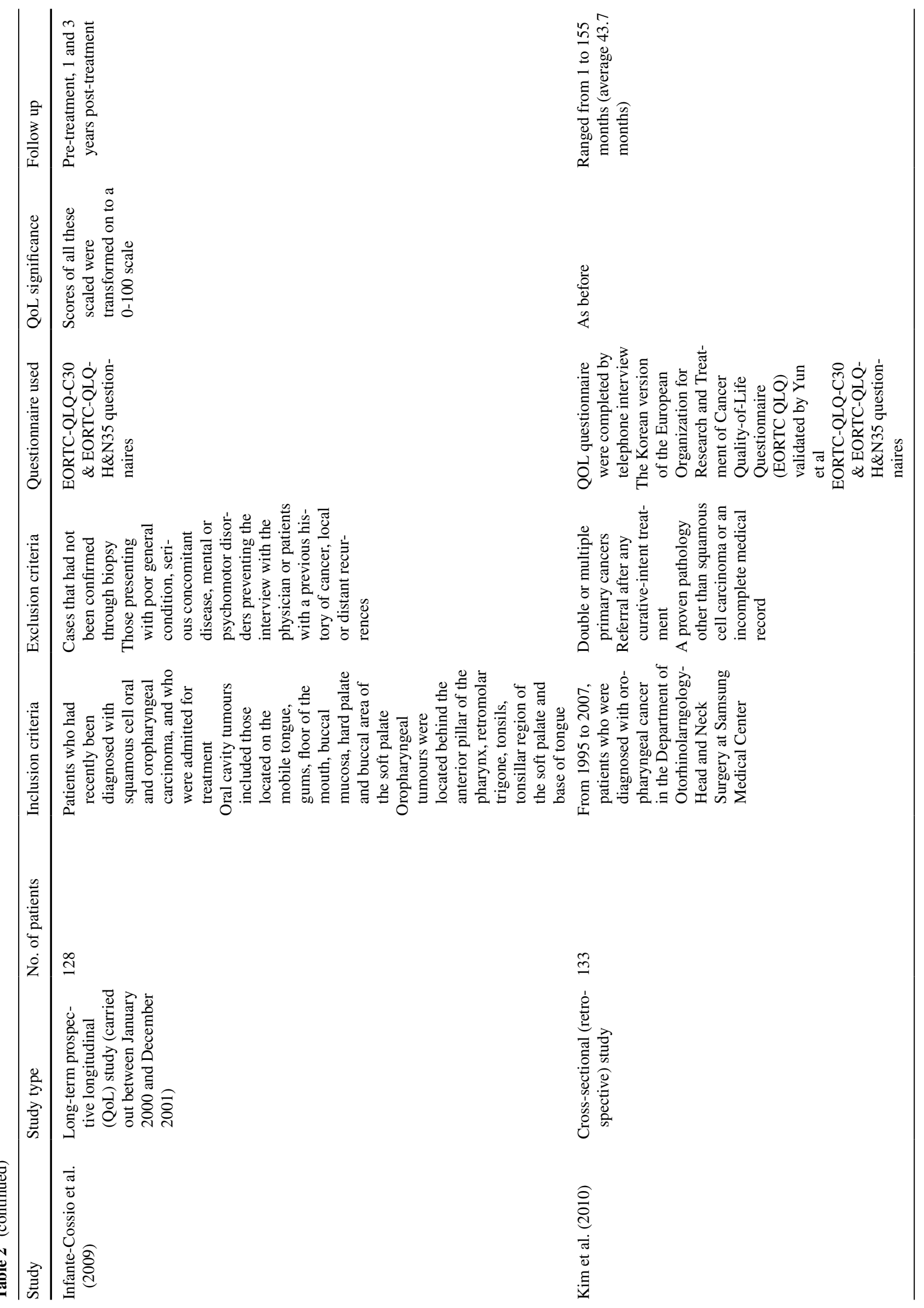


$(p=0.03)$ [43]. Comparison between patients receiving any kind of radiation therapy \pm surgery and those treated with surgery alone, radiation therapy leads to significantly more problems with mouth opening $(p=0.001)$ [43].

Kim et al. investigated OPC cancer patients and found that there was no significant difference in mouth opening between the surgery-based group and the RT-based group $(p=0.9024)[7]$.

Boscolo-Rizzo et al. analysed OPC patients and found that the chemoradiotherapy (CRT) group reported significantly greater problems with mouth opening $(p=0.036$, mean difference 18.1) when compared with those who had surgery + postoperative RT [44].

Postoperative radiotherapy had a significant correlation to impact mouth opening on the EORTC QLQ-H\&N35 symptom scale among patients who had undergone marginal or segmental mandibular resection for oral and oropharyngeal squamous cell carcinoma (OSCC) $(p=0.003$, $p \leq 0.003$ ) [42].

Patients who had recently been diagnosed with squamous cell oral and oropharyngeal carcinoma, who underwent surgery combined with adjuvant RT and chemotherapy presented worse evaluations of QoL, more affectation of less oral opening compared to those who had surgery alone [45].

\section{Discussion}

Subjective quality-of-life measures are a concurrent part of modern surgical practice. Ideally, these questionnaires should be validated [27]. Other QOL questionnaires, such as the mandibular function impairment questionnaire (MFIQ) [21] and Performance Status Scale (PSS) [28], have not been validated, while Van Der Molen et al. used a study-specific questionnaire for QOL evaluation [46].

The European Organization for Research and Treatment of Cancer have shown a great interest in module development. A module may assess (1) the disease symptoms related to a specific tumour site (e.g., abdominal pain in colorectal cancer), (2) the side-effects relating to a specific treatment (e.g., radiotherapy-induced skin problems), or (3) additional QoL domains affected by the disease of treatment (e.g., sexuality, body image and future perspective) [47]. The developmental process of these questionnaires is subjected to internal peer review to ensure uniformly highquality modules. These sets of questionnaires have undergone three phases of module development, psychometric performance, and cross-cultural validity [47]. The multinational, cross-cultural, and multidisciplinary composition of the EORTC study group have enabled crucial scientific and cultural input to the development of the modules [47]. These are among the reasons that the EORTC QLQ sets of questionnaires have been extensively used in studies to measure trismus in head and neck cancer. The LORQ questionnaire has showed promising abilities to assess oral rehabilitation in patients with oral and oropharyngeal cancer, showing good construct validity and reliability in a pilot study [48]. It was used alongside the UWQOL and the EORTC QLQ questionnaires to determine its validity and reliability [48]. However, the study size was small and future studies might have to look into analysing this questionnaire with a larger cohort [48].

Although the EORTC QLQ-C30 and the QLQ-H\&N35 have been used more frequently, it is undeniable that the GTQ questionnaire is a potential successor as it was found to be useful in identifying patients' change in functional mouth opening over time [20]. As for now, the EORTC QLQ sets of questionnaires are the set standard for a thorough, valid, and reliable method to determining quality of life in head and neck cancer patients.

\section{Surgery}

Nonsurgical treatments, deemed less invasive, are assumed to lead to better QoL outcomes [44, 49]. However, Ryzek et al. using the EORTC questionnaire for mouth opening reported higher scores among surgery-only patients when compared to surgery plus RT or any adjuvant therapy [27].

Patients undergoing surgery \pm RT had less restricted mouth opening, using subjective measures, than the nonsurgically treated patients. In this group, primary surgical resection achieved the highest QoL score in the head and neck specific EORTC QLQ-H\&N35 module of the three treatment modalities [43]. However, careful interpretation is required as these patients presented with a lower initial tumour stage than those treated with primary radiotherapy or surgery plus adjuvant radiotherapy [43]. We have included Trans Oral Robotic Surgery (TORS) in our search, but it did not yield any trismus related PROMs or studies.

\section{Radiotherapy}

The EORTC QLQ-C30 and H\&N35 used to subjectively measure trismus revealed that non-irradiated patients had significantly less trouble with mouth opening compared to those treated with either primary or postoperative radiation therapy [43]. However, mouth opening was not significantly different between surgery-based and RT-based treatment in a study by Kim et al. who also used the EORTC QLQ-C30 and H\&N35 [7, 43]. 
Fig. 2 Algorithm for measuring trismus in future studies. Assessment to include the following: Objective use callipers or similar instrument to measure the inter-incisor distance. Document in patient notes and/or database. Subjective use questionnaire (EORTC QLQ-C-30 and EORTC QLQ-H\&N35) to score patients' reported perception of trismus. Questionnaire added to patients' notes and/or database

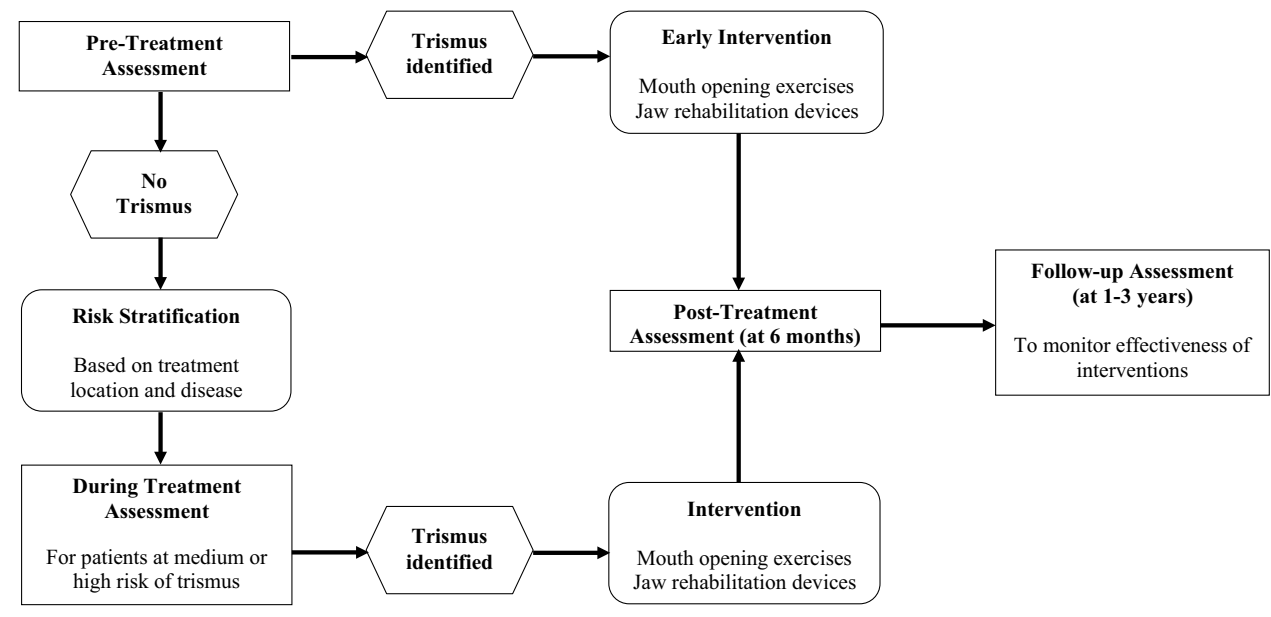

\section{Chemotherapy}

Positive predictive factors of trismus include treatment with concurrent chemoradiotherapy [50]. The CRT treatment group reported significantly greater problems with trismus than surgical patients using EORTC QLQ-C30 \& QLQ-HV35 [44], where patients with T3-4 oropharyngeal cancer after surgery plus postoperative RT (26 patients) were compared to versus CCRT (31 patients). Conflicting results, using the subjective measures do arise, as seen by Payakachat et al. when using the EORTC QLQ-H\&N35, the median score differences on the open mouth item were significantly higher in the Surgery + RT group when compared with the CRT group [51].

\section{Multimodality treatment}

Few studies compare QOL outcomes in different treatment modalities of oropharyngeal cancers [52, 53]. The studies that do look at QOL outcomes do not specifically look into the aspect of mouth opening [52, 53]. Only Infante-Cossio et al. showed that patients who underwent surgical treatment combined with adjuvant radiotherapy and chemotherapy generally showed a worse score for mouth opening, needing a longer recovery time compared to surgery alone and surgery plus RT [45].

\section{Tumour staging}

One study showed that patients in stages III and IV had a worse evaluation of their state of health and QoL, showing a higher incidence of pain, tiredness, less appetite, more swallowing problems, speech problems and problems with social contacts and eating in public, limited oral opening cough, weight loss, and more analgesia consumption [45]. There is a significant association between tumour staging and QoL problems [41, 54-56]. Patients with early stage cancer showed better overall QoL, both at the beginning and after 1 year, than those at more advanced stages [45].

\section{Trismus study design}

As per guidelines, mouth opening should be measured throughout treatment. For the purposes of future research, we have devised an algorithm for future studies to demonstrate when to measure for trismus at the various stages of the patients' treatment and follow-up (Fig. 2). This algorithm includes minimum points for trismus assessment and data capture, i.e., at the time of diagnosis, post treatment, and after rehabilitation. We suggest using the EORTC set of questionnaires, which are the EORTC QLQ-C30 and the EORTC QLQ-H\&N35 as they were the most established and were used in the studies included in our systematic review. We suggest a further trismus assessment 1 year post-treatment as studies report no further changes in health-related quality of life after the first postoperative year [57]. We suggest that patients should be followed up for at least 3 years [45].

\section{Limitations}

Our systematic review of subjective outcome measures contained studies with small cohorts. Furthermore, the existence of numerous confounding factors when comparing different treatment modalities, which cannot be eliminated are an ongoing limitation while utilising subjective trismus measures [27]. Other limitations include lack of information on the relationship between objective and subjective measures in most studies, hence not being able to ascertain the reliability of other questionnaires in 
practice. There are also variations between questionnaires in how they determine QoL with regard to the type of questions asked and also indirect parameters that suggest or can cause difficulty in mouth opening, such as chewing, speech, and pain. Some studies also focused on certain types of cancer, such as oropharyngeal cancer, while others took into account generally all cancers of the head and neck.

\section{Conclusion}

Subjective measures were utilised to measure post-operative trismus successfully. While cure rates are given the greatest priority and treatment if guided by the UICC grade, subjective measures, such as patient, reported outcome measures (PROMs) and QoL questionnaires are important and useful tools for assessing patient wellbeing. This is particularly important in head and neck cancer, where the treatment modalities result in equal effectiveness.

These subjective measures, QoLs and PROMs, are also of use in monitoring side effects of treatment, e.g., trismus, so that early intervention can be implemented to treat or prevent progression. This manuscript demonstrates that future head and neck cancer treatment studies must incorporate functional measurements together with QOL measurements, because functional improvements alone do not correlate to a perceived improvement in trismus outcomes according to patients [7]. We envisage future studies prospectively performing objective and subjective measures of mouth opening prior, during and after treatment. Subjective measures may be early indicators of developing trismus, which would have implications for early intervention.

\section{Compliance with ethical standards}

Funding The authors did not receive any financial support or other external help in preparing this manuscript.

Conflict of interest The authors declare that they have no conflict of interest.

Ethical approval This article does not contain any studies with human participants or animals performed by any of the authors.

Open Access This article is distributed under the terms of the Creative Commons Attribution 4.0 International License (http:// creativecommons.org/licenses/by/4.0/), which permits unrestricted use, distribution, and reproduction in any medium, provided you give appropriate credit to the original author(s) and the source, provide a link to the Creative Commons license, and indicate if changes were made.

\section{References}

1. Vokes EE, Weichselbaum RR, Lippman SM, Hong WK (1993) Head and neck cancer. N Engl J Med 3:184-194

2. Tobias JS (1994) Cancer of the head and neck. BMJ 308:961-966

3. Argiris A, Karamouzis MV, Raben D, Ferris RL (2008) Head and neck cancer. Lancet (London, England) 371:1695-1709

4. Licitra L, Bernier J, Grandi C, Merlano M, Bruzzi P, Lefebvre J-L (2002) Cancer of the oropharynx. Crit Rev Oncol Hematol 41:107-122

5. Jemal A, Bray F, Center MM, Ferlay J, Ward E, Forman D (2011) Global cancer statistics. CA Cancer J Clin 61:69-90

6. SEER Cancer Statistics Review, 1975-2013. http://seer.cancer. gov/csr/1975_2013/. Accessed 23 Oct 2016

7. Kim TW, Youm HY, Byun H, Son YI, Baek CH (2010) Treatment outcomes and quality of life in oropharyngeal cancer after surgery-based versus radiation-based treatment. Clin Exp Otorhinolaryngol 3:153-160

8. Weber C, Dommerich S, Pau HW, Kramp B (2010) Limited mouth opening after primary therapy of head and neck cancer. Oral Maxillofac Surg 14:169-173

9. Wang C-J, Huang E-Y, Hsu H-C, Chen H-C, Fang F-M, Hsiung C-Y (2005) The degree and time-course assessment of radiationinduced trismus occurring after radiotherapy for nasopharyngeal cancer. Laryngoscope 115:1458-1460

10. Jager-Wittenaar H, Dijkstra PU, Vissink A, van Oort RP, Roodenburg JLN (2009) Variation in repeated mouth-opening measurements in head and neck cancer patients with and without trismus. Int J Oral Maxillofac Surg 38:26-30

11. Lee L-Y, Chen S-C, Chen W-C, Huang B-S, Lin C-Y (2015) Postradiation trismus and its impact on quality of life in patients with head and neck cancer. Oral Surg Oral Med Oral Pathol Oral Radiol 119:187-195

12. Vissink a, Jansma J, Spijkervet FKL, Burlage FR, Coppes RP (2003) Oral sequelae of head and neck radiotherapy. Crit Rev Oral Biol Med 14:199-212

13. Ichimura K, Tanaka T (1993) Trismus in patients with malignant tumours in the head and neck. J Laryngol Otol 107:1017-1020

14. Goldstein M, Maxymiw WG, Cummings BJ, Wood RE (1999) The effects of antitumor irradiation on mandibular opening and mobility: a prospective study of 58 patients. Oral Surg Oral Med Oral Pathol Oral Radiol Endod 88:365-373

15. Dreizen SA, Dally TE, Drane JB, Brown LR (1977b) Oral complications of cancer radiotherapy. Postgr Med 61:85-92

16. Engelmeier RL, King GE (1983) Complications of head and neck radiation therapy and their management. J Prosthet Dent 49:514-522

17. Vissink A, Burlage FR, Spijkervet FKL, Jansma J, Coppes RP (2003) Prevention and treatment of the consequences of head and neck radiotherapy. Crit Rev Oral Biol Med 14:213-225

18. Dhanrajani PJ, Jonaidel O (2002) Trismus: aetiology, differential diagnosis and treatment. Dent Update 29:88-92, 94.

19. Dworkin SF, Huggins KH, LeResche L, Von Korff M, Howard J, Truelove E, Sommers E (1990) Epidemiology of signs and symptoms in temporomandibular disorders: clinical signs in cases and controls. J Am Dent Assoc 120:273-281

20. Pauli N, Johnson J, Finizia C, Andréll P (2013) The incidence of trismus and long-term impact on health-related quality of life in patients with head and neck cancer. Acta Oncol 52:1137-1145

21. Dijkstra PU, Huisman PM, Roodenburg JLN (2006) Criteria for trismus in head and neck oncology. Int J Oral Maxillofac Surg 35:337-342

22. Kamstra JI, Dijkstra PU, van Leeuwen M, Roodenburg JLN, Langendijk JA (2015) Mouth opening in patients irradiated for 
head and neck cancer: a prospective repeated measures study. Oral Oncol 51:548-555

23. Lee R, Slevin N, Musgrove B, Swindell R, Molassiotis A (2012) Prediction of post-treatment trismus in head and neck cancer patients. Br J Oral Maxillofac Surg 50:328-332

24. Van Der Molen L, Heemsbergen WD, De Jong R, Van Rossum MA, Smeele LE, Rasch CRN, Hilgers FJM (2013) Dysphagia after chemoradiotherapy Dysphagia and trismus after concomitant chemo-Intensity-Modulated Radiation Therapy (chemoIMRT) in advanced head and neck cancer; Dose-effect relationships for swallowing and mastication structures. Radiother Oncol 106:364-369

25. Scott B, D’Souza J, Perinparajah N, Lowe D, Rogers SN (2011) Longitudinal evaluation of restricted mouth opening (trismus) in patients following primary surgery for oral and oropharyngeal squamous cell carcinoma. Br J Oral Maxillofac Surg 49:106-111

26. Scott B, Butterworth C, Lowe D, Rogers SN (2008) Factors associated with restricted mouth opening and its relationship to health-related quality of life in patients attending a Maxillofacial Oncology clinic. Oral Oncol 44:430-438

27. Ryzek D-F, Mantsopoulos K, Künzel J, Grundtner P, Zenk J, Iro H, Psychogios G (2014) Early stage oropharyngeal carcinomas: comparing quality of life for different treatment modalities. Biomed Res Int 2014:421964

28. Teguh DN, Levendag PC, Voet P, van der Est H, Noever I, de Kruijf W, van Rooij P, Schmitz PIM, Heijmen BJ (2008) Trismus in patients with oropharyngeal cancer: relationship with dose in structures of mastication apparatus. Head Neck 30:622-630

29. Stegenga B, L. G. M. de Bont, de Leeuw GB R (1993) Assessment of mandibular function impairment associated with temporomandibular joing osteoarthrosis and internal derangement. J Orofac Pain 7:183-195

30. Pace-Balzan A, Cawood JI, Howell R, Butterworth CJ, Lowe D, Rogers SN (2006) The further development and validation of the Liverpool Oral Rehabilitation Questionnaire: a cross-sectional survey of patients attending for oral rehabilitation and general dental practice. Int J Oral Maxillofac Surg 35:72-78

31. Hutcheson, Katherine A, Lewin JS (2012) Functional outcomes after chemoradiotherapy of laryngeal and pharyngeal cancers. Curr Oncol Rep 14:158-165

32. List MA, D'Antonio LL, Cella DF, Siston A, Mumby P, Haraf D, Vokes E (1996) The performance status scale for head and neck cancer patients and the functional assessment of cancer therapy-head and neck scale: a study of utility and validity. Cancer 77:2294-2301

33. Duke RL, Campbell BH, Indresano AT, Eaton DJ, Marbella AM, Myers KB, Layde PM (2005) Dental status and quality of life in long-term head and neck cancer survivors. Laryngoscope 115:678-683

34. Aaronson NK, Ahmedzai S, Bergman B, Bullinger M, Cull A, Duez NJ, Filiberti A, Flechtner H, Fleishman SB, Haes JCJM d., Kaasa S, Klee M, Osoba D, Razavi D, Rofe PB, Schraub S, Sneeuw K, Sullivan M, Takeda F (1993) The European Organization for Research and Treatment of Cancer QLQ-C30: a quality-of-life instrument for use in international clinical trials in oncology. JNCI J Natl Cancer Inst 85:365-376

35. Huguenin PU, Taussky D, Moe K, Meister A, Baumert B, Lütolf UM, Glanzmann C (1999) Quality of life in patients cured from a carcinoma of the head and neck by radiotherapy: The importance of the target volume. Int J Radiat Oncol Biol Phys 45:47-52

36. Bjordal K, Hammerlid E, Ahlner-Elmqvist M, de Graeff A, Boysen M, Evensen JF, Biörklund A, de Leeuw JR, Fayers PM, Jannert M, Westin T, Kaasa S (1999) Quality of life in head and neck cancer patients: validation of the European Organization for Research and Treatment of Cancer Quality of Life Questionnaire-H\&N35. J Clin Oncol 17:1008-1019
37. Ng W-MR, Wei IW (2006) Quality of life of patients with recurrent nasopharyngeal carcinoma treated with nasopharyngectomy using the maxillary swing approach. Arch Otolaryngol Head Neck Surg 132:309-316

38. Bjordal K, Kaasa S (1992) Psychometric validation of the EORTC Core Quality of Life Questionnaire, 30-item version and a diagnosis-specific module for head and neck cancer patients. Acta Oncol 31:311-321

39. Bjordal K, AhlnerElmqvist M, Tollesson E, Jensen AB, Razavi D, Maher EJ, Kaasa S: Development of A European-Organization-For-Research-And-Treatment-Of-Cancer (Eortc) questionnaire module to be used in quality-of-life assessments in head and neck-cancer patients. Acta Oncologica 1994:879-885

40. Steiner F, Evans J, Marsh R, Rigby P, James S, Sutherland K, Wickens R, Nedev N, Kelly B, Tan ST (2015) Mouth opening and trismus in patients undergoing curative treatment for head and neck cancer. Int J Oral Maxillofac Surg 44:292-296

41. Rogers SN, Lowe D, Patel M, Brown JS, Vaughan ED (2002) Clinical function after primary surgery for oral and oropharyngeal cancer: an 11-item examination. Br J Oral Maxillofac Surg 40:1-10

42. Van Cann EM, Dom M, Koole R, Merkx MAW, Stoelinga PJW (2005) Health related quality of life after mandibular resection for oral and oropharyngeal squamous cell carcinoma. Oral Oncol 41:687-693

43. Tschudi D, Stoeckli S, Schmid S (2003) Quality of life after different treatment modalities for carcinoma of the oropharynx. Laryngoscope 113:1949-1954

44. Boscolo-Rizzo P, Stellin M, Fuson R, Marchiori C, Gava A, Da Mosto MC (2009) Long-term quality of life after treatment for locally advanced oropharyngeal carcinoma: surgery and postoperative radiotherapy versus concurrent chemoradiation. Oral Oncol 45:953-957

45. Infante-Cossio $\mathrm{P}$, Torres-Carranza E, Cayuela A, Hens-Aumente E, Pastor-Gaitan P, Gutierrez-Perez JL (2009) Impact of treatment on quality of life for oral and oropharyngeal carcinoma. Int J Oral Maxillofac Surg 38:1052-1058

46. Van Der Molen L, Van Rossum MA, Burkhead LM, Smeele LE, Rasch CRN, Hilgers FJM (2011) A randomized preventive rehabilitation trial in advanced head and neck cancer patients treated with chemoradiotherapy: feasibility, compliance, and short-term effects. Dysphagia 26:155-170

47. Sprangers MAG, Cull A, Groenvold M, Bjordal K, Blazeby J, Aaronson NK (1998) The European Organization for Research and Treatment of Cancer approach to developing questionnaire modules: an update and overview. Qual Life Res 7:291-300

48. Pace-Balzan A, Cawood JI, Howell R, Lowe D, Rogers SN (2004) The liverpool oral rehabilitation questionnaire: a pilot study. J Oral Rehabil 31:609-617

49. Tribius S, Bergelt C: Intensity-modulated radiotherapy versus conventional and 3D conformal radiotherapy in patients with head and neck cancer: Is there a worthwhile quality of life gain? Cancer Treat Rev 2011:511-519

50. Jeremic G, Venkatesan V, Hallock A, Scott D, Hammond A, Read N, Franklin J, Yoo J, Fung K (2011) Trismus following treatment of head and neck cancer. J Otolaryngol Head Neck Surg 40:323-329

51. Payakachat N, Ounpraseuth S, Suen JY (2013) Late complications and long-term quality of life for survivors ( $>5$ years) with history of head and neck cancer. Head Neck 35:819-825

52. Broglie M a., Soltermann A, Haile SR, Röösli C, Huber GF, Schmid S, Stoeckli SJ (2013) Quality of life of oropharyngeal cancer patients with respect to treatment strategy and p16-positivity. Laryngoscope 123:164-170

53. Tschiesner U, Schuster L, Strieth S, Harréus U (2012) Functional outcome in patients with advanced head and neck cancer: 
surgery and reconstruction with free flaps versus primary radiochemotherapy. Eur Arch Otorhinolaryngol 269:629-638

54. Graeff A d., JR d. L, WJ R, GJ H, GH B, JA W (1999) A prospective study on quality of life of patients with cancer of the oral cavity or oropharynx treated with surgery with or without radiotherapy. Oral Oncol 35:27-32

55. de Graeff a, de Leeuw JR, Ros WJ, Hordijk GJ, Blijham GH, Winnubst J a (2000) Long-term quality of life of patients with head and neck cancer. Laryngoscope 110:98-106
56. Hammerlid E, Bjordal K, Ahlner-Elmqvist M, Boysen M, Evensen JF, Biorklund A, Jannert M, Kaasa S, Sullivan M, Westin T (2001) A prospective study of quality of life in head and neck cancer patients. Part I: at diagnosis. Laryngoscope 111(4 Pt 1):669-680

57. Rogers SN, Hannah L, Lowe D, Magennis P (1999) Quality of life 5-10 years after primary surgery for oral and oro-pharyngeal cancer. J Craniomaxillofac Surg 27:187-191 